\title{
Festsetzung regionalpolitischer Prioritäten im Schweizer Alpenraum auf der Basis regionaler Input-Output-Tabellen - ein Überblick
}

\author{
Benjamin Buser, Gianluca Giuliani, Peter Rieder, \\ Zürich
}

\section{Wachstumsschwächen im Berggebiet}

Die Schweiz hatte in den vergangenen zehn Jahren das geringste Wirtschaftswachstum aller 29 OECDStaaten. Gleichzeitig haben die Wachstumsrückstände der Regionen im Alpenraum gegenüber den nationalen Zentren weiter zugenommen (OECD 2002). Diese Zunahme räumlicher Disparitäten erfolgte trotz kostenintensiven sektoral- und regionalpolitischen Massnahmen für das Berggebiet. Die bestehenden regionalpolitischen Instrumente werden zunehmend als wenig effektiv erkannt (Thierstein \& BeHRENDT 2001). Daher findet zurzeit eine Neukonzeption der Förderung und Unterstützung wirtschaftlich bedrohter Wirtschaftszweige und Landesgegenden statt (EXPERTENKOMMISSION 2003). Bund und Kantone sind mittels Bundesverfassung auch in Zukunft hierzu angehalten. Eine Neukonzeption regionalpolitischer Instrumente erhöht den Bedarf an Kenntnissen über regionalwirtschaftliche Zusammenhänge. Insbesondere die Festsetzung regionaler Prioritäten bei unterschiedlichen wachstumspolitischen Strategien stellt ein Hauptproblem dar. Ohne fundierte Kenntnisse über die tatsächlichen wirtschaftlichen Ist-Zustände ist die Herleitung effizienter und effektiver Wachstumsstrategien hin zu wünschenswerten Soll-Zuständen schwierig. Beide Beurteilungen sind quantitativ allerdings nur mit Rückgriff auf Kennzahlen des regionalen Wertschöpfungssystems möglich.

Im Rahmen der Neuorientierung der Regionalpolitik hat eine Expertenkommission des Bundes zwei wirtschaftspolitische Handlungsleitlinien zur Vision der Regionalpolitik erklärt (EXPERTENKOMMISSION 2003):

1. Erhöhung der Innovationsfähigkeit in den Regionen,

2. bessere Vernetzung regionaler Wertschöpfungssysteme.

Darüber hinaus wird eine regionale Wirtschaftspolitik gefordert, welche von starken nationalen Zentren und dazugehörigen Randregionen ausgeht. Während Unternehmen in nationalen Zentren ihre Produktion auf international stetig wachsende Märkte ausrichten, konzentrieren sich die Unternehmen in Randregionen primär auf die Nachfrage in den nationalen Zentren.
Die regionalen Implikationen und Umsetzungsansätze wurden durch die Expertenkommission wenig konkretisiert. Dieser Artikel nimmt sich daher der folgenden zwei Fragen an:

1. Wie stellt sich der regionale Ist-Zustand in ausgewählten Regionen des Schweizer Alpenraums heute dar und welche Wachstumsschwächen gilt es hin zu den geforderten Soll-Zuständen mit Wirtschaftswachstum über lange Frist zu überwinden?

2. Wie können die identifizierten Wachstumsschwächen überwunden und effizient wie auch effektiv anhaltendes Wirtschaftswachstum herbeigeführt werden bzw. welchen Beitrag kann eine Regionalpolitik hierfür leisten?

Der Artikel beantwortet diese Fragen, indem zuerst die methodischen Grundlagen der Untersuchung erläutert werden. Anschliessend folgen einige Ausführungen zu den wichtigsten makroökonomischen Theorien der Regionalentwicklung. Im empirischen Teil werden Untersuchungsresultate aus ausgewählten Regionen im Schweizer Alpenraum präsentiert. Abschliessend werden ein wachstumspolitisches Modell und dessen Integration in die Regionalpolitik diskutiert.

\section{Grundlagen der regionalen Input-Output-Analyse}

Ein empirischer Ansatz zur Untersuchung regionaler Wirtschaftskreisläufe ist die Input-Output-Analyse (Holub \& Schnabl 1994). Hierfür werden die wirtschaftlichen Verflechtungen aller Unternehmen sowie privater und öffentlicher Haushalte einer Region in einer Kreuztabelle, der sogenannten Input-OutputTabelle (IOT abgekürzt) erfasst. Die Kreuztabelle entspricht der Abbildung der Geldflüsse. In der Tabelle wird - nach Wirtschaftsgruppen zusammengefasst - ersichtlich, wo, bei wem und in welchem Umfang gegenseitig Inputs bezogen beziehungsweise Outputs abgesetzt werden. Zentral im Wirtschaftskreislauf sind hierbei die Vorleistungsbeziehungen zwischen den Branchen.

Der Beitrag stellt eine Zusammenfassung des Buches von B. BUSER (2005): Regionale Wirtschaftskreisläufe und regionale Wachstumspolitik. Regionalpolitische Prioritäten für unterschiedliche Regionen im Schweizer Alpenraum auf der Basis regionaler Input-Output Tabellen $=$ Berichte aus der Volkswirtschaft, Aachen, Shaker Verlag, dar. 
Als Vorleistungen werden Produkte und Dienstleistungen bezeichnet, welche im Rahmen des Produktionsprozesses eine Branche an eine andere Branche liefert. Neben den Vorleistungslieferungen werden Produkte und Dienstleistungen sowohl an die regionale Endnachfrage der öffentlichen und privaten Haushalte geliefert als auch exportiert und importiert. In einer IOT werden Importe und Exporte nach Herkunfts- beziehunsweise Bestimmungsregionen zusammengefasst.

Die IOT erfasst das Volumen sämtlicher Geldflüsse eines Jahres und bildet das regionalwirtschaftliche Gleichgewicht $a b$. Aus der richtigen Gruppierung von regionalen Umsätzen und regionalen Vorleistungsbezügen leitet sich das regionale Bruttoinlandprodukt ab. Dieses wird Bruttoregionalprodukt (BRP) genannt und lässt bei Division durch die Beschäftigten oder durch die Bevölkerung regionale Vergleiche der Wirtschaftskraft zu. Unterschiedliche Wirtschaftskraft ist Ausdruck entstehender Disparitäten.

Neben den deskriptiven Eigenschaften besitzt eine regionale IOT nützliche analytische Eigenschaften. Dem Input-Output-Ansatz liegt folgende Vorstellung über Wirtschaftskreisläufe zu Grunde: Erfährt eine Branche A eine höhere Nachfrage mit Umsatzerhöhungen um eine Einheit, wird dies direkter Effekt genannt. Für die Produktion einer zusätzlichen Einheit benötigt die Branche A zusätzliche Vorleistungen und Arbeitsleistung, welche sie z.B. bei den Branchen B, C oder bei den privaten Haushalten bezieht. Die zusätzlich benötigten Produktionsmittel stehen in einem konstanten Verhältnis zur ursprünglichen Nachfrageänderung (das heisst, es gelten linear-limitationale Produktionsfunktionen). Für die Branchen B, C beispielsweise erhöht sich ihrerseits die Nachfrage und entsprechend der Bedarf an Vorleistungen. Es setzt eine unendliche Reaktionskette ein, wobei die zusätzlichen Vorleistungsbezüge gegen Null streben.

Durch die erhöhte Arbeitsnachfrage in jedem Schritt der Reaktionskette erhöhen sich Einkommen und Konsummöglichkeiten. Dies löst eine weitere Abfolge von unendlichen Nachfrageänderungen mit immer kleineren nominalen Beträgen aus. Sämtliche Effekte, resultierend aus dieser zusätzlichen Vorleistungsnachfrage werden «indirekte» Effekte, die entsprechenden Effekte aus den zusätzlichen Konsumausgaben «induzierte» Effekte genannt.

Direkter Effekt, indirekte Effekte und induzierte Effekte können zu einem Gesamteffekt zusammengezählt werden, wobei vor allem die Effekte interessieren, welche tatsächlich innerhalb und nicht durch den Import von Vorleistungen ausserhalb einer Untersuchungsregion anfallen. Das Verhältnis von Gesamteffekt zu direktem Effekt ergibt einen Nachfragemul- tiplikator. Dieser Nachfragemultiplikator ist für jede Branche unterschiedlich und kann durch die Summe einer unendlichen geometrischen Reihe mathematisch bestimmt werden (Holub \& Schnabl 1994). Ist ein Nachfragemultiplikator für eine Branche bekannt, lässt sich berechnen, welche relativen Effekte branchenspezifische Nachfrageänderungen beispielsweise auf sämtliche Branchenumsätze, den regionalen Gesamtumsatz, die regionale Wertschöpfung, die regionale Arbeitsnachfrage oder ausbezahlte Arbeitseinkommen haben.

Die Stabilität dieser Nachfragemultiplikatoren über den zeitlichen Verlauf hinweg ist abhängig vom wirtschaftlichen Strukturwandel. Ist dieser in einer Region bereits weit fortgeschritten und langsam, dürfen Nachfragemultiplikatoren über lange Zeit hinweg als stabil angenommen werden. Die Unternehmen finden regional ein konstantes Angebot an Vorleistungen vor. Läuft hingegen der Strukturwandel, verbunden mit Eintritt und Austritt von Unternehmen und Branchen in eine Region, schnell ab, stellen Nachfragemultiplikatoren lediglich Momentaufnahmen dar. Die Effekte von schnell ablaufenden Strukturanpassungen können nur bedingt, mittels vergangenheitsbezogenen Strukturanpassungskoeffizienten, in das Kreislaufmodell implementiert werden. In vielen Bergregionen ist der Strukturwandel heute langsam oder gar blockiert, womit sich regionale Wachstumsschwächen partiell erklären lassen.

\section{Makroökonomische Verankerung}

Im Zentrum aller regionalen Wachstumstheorien steht die Frage, welche Prozesse langfristig zu einem positiven Wachstum der regionalen Wertschöpfung führen. Die Entwicklung z.B. von Beschäftigung, Einkommen, Kapital wird letztlich immer nur als Folge des Wertschöpfungswachstums betrachtet (siehe auch KoPAINSKY 2005; Kopainsky \& Rieder in diesem Heft).

Die meisten Wachstumstheorien basieren entweder auf der klassischen oder aber auf der keynsianischen Theorie makroökonomischer Zusammenhänge.

Für die klassische Makroökonomie gilt, dass Märkte immer nach einem allokationseffizienten Gleichgewicht streben (MENGHETTI \& ZimmermanN 1996). Als Folge rationaler individueller Nutzenmaximierung werden Produktionsfaktoren immer an den Produktionsstandort verschoben, wo die höchste Grenzproduktivität erreicht wird. Die Faktormobilität ist gegeben, sofern vollständige Informationen, vollständige Konkurrenz und keine Transaktionskosten existieren. Sämtliche Produktionsfaktoren werden hierdurch allokationseffizient eingesetzt, wodurch eine Regionalwirtschaft wachsen kann. 
Reale Wachstumsschwächen bestehen darin, dass Produktionsfaktoren durch Marktunvollkommenheiten suboptimal und ineffizient eingesetzt werden. Beheben sich diese Unvollkommenheiten (beispielsweise Hindernisse der Faktormobilität oder künstliche Transaktionskosten) längerfristig nicht von selbst, ist der Staat zum Eingriff legitimiert. Ebenso darf der Staat intervenieren, wenn die sich einstellende Faktorallokation gesellschaftlichen Verteilungszielen entgegenläuft. Ansonsten gilt für den Staat wirtschaftspolitische Abstinez (Hahne \& von Stackelberg 1994).

Die keynsiansche Makroökonomie hat sich als Gegenpol zur klassischen Theorie etabliert. Im Vordergrund dieser Theorien stehen nicht Angebot und Nachfrage nach Produktionsfaktoren, sondern die reale Güternachfrage (Menghetti \& ZimmermanN 1996). Das Niveau der Güternachfrage wirkt unmittelbar auf die Auslastung der Produktionsfaktoren, wodurch diese über längere Zeiträume unausgelastet sein können. Unterauslastung von Produktionsfaktoren führt zu Schwankungen in der wirtschaftlichen Entwicklung und letztlich zu Wohlfahrtsverlusten. Im Gegensatz zur klassischen Makroökonomie bestehen immer Marktunvollkommenheiten, was beispielsweise die Existenz von Monopolen ermöglicht. Der Staat wird niemals in der Lage sein, diese Unvollkommenheiten zu eliminieren. Einzig die Erhöhung der Realnachfrage überwindet suboptimale Zustände und löst neue Wachstumsprozesse mit Wohlfahrtsgewinnen aus. Dies legitimiert den Staat jederzeit zu einer intervenistischen Wirtschaftspolitik, welche auf Staatskonsum aufbaut.

Die vorliegende Untersuchung misst der klassischen Theorie hohes Gewicht bei, da diese die Grundlage für die empirische Modellierung der Wirtschaftskreisläufe bildet. Anderseits zeigt die reale Situation regelmässig eine bessere Validität der keynsianischen Annahmen. Beispielsweise ist die Schweizer Wirtschaftspolitik klassisch konzipiert und strebt Wachstum durch bessere Produktionsbedingungen an. Zumeist beschränkt sich jedoch die Wachstumswirkung ausschliesslich auf die getätigte Investition. Werden Wachstumsschwächen dadurch nur kurzfristig überwunden, muss eine Wirtschaftspolitik tatsächlich als keynsianisch angesehen werden.

\section{Fünf regionale Wirtschaftskreisläufe}

Regionale Input-Output-Tabellen erfordern eine enorme Datenfülle, wovon nur ein geringer Anteil über statistische Ämter zu beziehen ist. Die Mehrheit der Daten zu Verflechtungen von Unternehmen und Haushalten müssen mittels ressourcenintensiven Datenerfassungen erhoben werden. Zwar bestehen mathe- matische Verfahren, um national erfasste Daten der volkswirtschaftlichen Gesamtrechnung auf Regionen aufzuschlüsseln (FURRER 2004). Die Validität solcher Daten ist jedoch ungenügend, da häufig nationale Mittelwerte regional zu wenig Gültigkeit besitzen. Die primäre Datensammlung bleibt vorerst der einzige Weg, um eine regionale IOT zu erstellen. Für die Regionen Val Bregaglia, Val Müstair, Urserntal, Vallemaggia und Brig-Visp-Naters wurden die notwendigen Daten erhoben (BUSER 2005).

Als einzige Methode, welche ausreichend Daten mit der erforderlichen Genauigkeit hervorbringt, hat sich eine flächendeckende, standardisierte Datenerhebung direkt am Unternehmensstandort erwiesen. Die persönliche Befragung erfordert gute Kenntnisse der Methode und des betrieblichen Buchhaltungswesens einerseits, anderseits müssen spezifische Charakteristiken von Produktionsprozessen erkannt und adäquat behandelt werden. Durch die parallele input- und output-seitige Datenerhebung kann mittels eines Differenzbereinigungsverfahrens eine verlässliche gesamtwirtschaftliche Identität zwischen Inputs und Outputs hergeleitet werden. Die Aggregation in wenige Branchen, welche sich unter anderem auch durch Gründe des Datenschutzes ergibt, erleichtert Erfassung und Berechnung der Identität, zwingt jedoch allzu oft zu pragmatischen Abgrenzungen.

Nachfolgend wird vergleichend ein Überblick über die vorgefundenen Wirtschaftsmuster und Wachstumsprozesse gegeben.

\subsection{Regionalisierungen zur Untersuchung}

Die fünf zu untersuchenden Regionen zeigen einerseits grosse Unterschiede in der vergangenen Bevölkerungsdynamik, anderseits in wirtschaftlichen Strukturen und Strukturentwicklungen (RIEDER et al. 2004). Ergänzt durch geographische Standorteigenschaften lassen sich die fünf Untersuchungsregionen zu drei Typen zusammenfassen:

- Die Regionen Val Bregaglia, Val Müstair und Urserntal sind geprägt durch eine periphere Lage zu den Zentren und starke Bevölkerungsabwanderung. Diese Regionen haben viele Arbeitsplätze verloren und sind existenziell vom landwirtschaftlichen Strukturwandel betroffen. Diesen Regionstyp kann man als klassische Entleerungsregion bezeichnen.

- Der städtische Raum Brig-Visp-Naters liegt in einem Alpenhaupttal und zeigt eine überdurchschnittliche Wachstumsdynamik. Umgeben von touristisch erschlossenen Seitentälern übernimmt die Region zentralörtliche Funktionen für eine übergeordnete Grossregion. Brig-Visp-Naters gilt als ein inneralpines Zentrum.

- Das Vallemaggia zeigt einerseits die Probleme der klassischen Entleerungsregion, anderseits exponen- 


\begin{tabular}{|c|c|c|c|c|c|}
\hline \multirow[t]{2}{*}{ Branche } & \multicolumn{5}{|c|}{ Multiplikator für die regionale Wertschöpfung } \\
\hline & Val Bregaglia* & Val Müstair** & Urserntal $* *$ & Brig-Visp-Naters* & Vallemaggia** \\
\hline Landwirtschaft & 0.58 & 0.86 & 0.84 & 0.91 & 0.82 \\
\hline Bergbau & -- & -- & -- & -- & 0.71 \\
\hline $\begin{array}{l}\text { Nahrungsmittelproduktion } \\
\text { Nahrungsmittelhandel }\end{array}$ & 0.25 & 0.42 & 0.40 & 0.80 & $\begin{array}{l}0.72 \\
0.30\end{array}$ \\
\hline Gewerbe & 0.44 & 0.53 & 0.60 & 0.66 & 0.75 \\
\hline Chemische Industrie & -- & -- & -- & 0.48 & - \\
\hline Bauwesen & 0.66 & 0.78 & 0.96 & 0.88 & 0.76 \\
\hline Energie, Wasser & 0.65 & 0.31 & 0.95 & 0.29 & 0.58 \\
\hline Handel, Reparatur & -- & 0.11 & -- & 0.77 & 0.46 \\
\hline $\begin{array}{l}\text { Tourismus } \\
\text { Gastgew erbe }\end{array}$ & 0.77 & 0.78 & $\begin{array}{l}0.67 \\
0.74\end{array}$ & 0.92 & 0.99 \\
\hline $\begin{array}{l}\text { Sonstige Dienstleistungen } \\
\text { Verkehr, Kommunikation } \\
\text { Immobilien, Informatik } \\
\text { Gesundheitswesen }\end{array}$ & 0.21 & 0.91 & 1.00 & $\begin{array}{l}0.98 \\
0.77 \\
0.55\end{array}$ & 0.80 \\
\hline Militär, Bundesstellen & -- & -- & 1.11 & - & -- \\
\hline Öffentlicher Sektor & 0.90 & 0.92 & 1.03 & 0.77 & 0.21 \\
\hline
\end{tabular}

* ohne induzierte Effekte, nur indirekte Effekte

${ }^{* *}$ mit indirekten und induzierten Effekten

Tab. 1: Wertschöpfungsmultiplikatoren für die Branchen der Untersuchungsregionen Multipliers of value added for all industries in the regions under investigation Multiplicateurs de création de valeur pour les branches des régions soumises à l'étude Quelle: Zusammenzug aus BUSER 2005

tielles Bevölkerungswachstum in Dörfern nahe des Talausganges in der Agglomeration von Locarno. Innerhalb der Region bestehen grosse Disparitäten mit einem Gradienten Talende - Agglomerationszentrum. Das Vallemaggia verkörpert eine zentrumsnahe Transformationsregion.

\subsection{Regionale Effizienz, Effektivität und Abhängigkeit} Nachfragemultiplikatoren können, in Abhängigkeit von der geographischen Herkunft von Vorleistungen und Arbeitskraft, hoch oder tief ausfallen. Ein hoher Multiplikator drückt aus, dass ein zusätzlicher Franken Nachfrage in einer Branche A viele zusätzliche Effekte in der Region auslöst. Das Verhältnis direkter (d.h. ursprünglicher) Effekt zu Gesamteffekt fällt günstig aus. Die Branche löst effizient regionales Wachstum aus.

Die Tabelle 1 zeigt die Multiplikatoren für die regionale Wertschöpfung. Diese können wie folgt gelesen werden: Wenn beispielsweise die Landwirtschaft des Val Bregaglia eine Nachfrageänderung um einen Franken erfährt, so verändert sich die totale regionale Wertschöpfung gesamthaft um 58 Rappen. Diese Änderung hat Gültigkeit sowohl mit positiven wie auch mit negativen Vorzeichen.

Auf Grund der Datenlage für das Val Bregaglia und die Region Brig-Visp-Naters konnten keine induzierten Effekte berechnet werden. Relative Vergleiche zwischen den Untersuchungsregionen und absolute Vergleiche innerhalb der Untersuchungsregionen sind jedoch jederzeit zulässig.

Die Wertschöpfungsmultiplikatoren zeigen regional grosse Unterschiede. Der einzelne Franken Umsatz in den sonstigen Dienstleistungen des Val Müstair löst mit 91 Rappen acht- bis neunmal mehr zusätzliche Wertschöpfung aus als der Handel mit nur 11 Rappen Wertschöpfung.

Eine hohe regionalwirtschaftliche Effizienz darf allerdings nicht per se mit Wirksamkeit gleichgesetzt werden. Die Branchen einer Region unterscheiden sich fundamental in der Höhe ihrer Umsätze und Wertschöpfungsanteile. Daraus ergeben sich auch unterschiedliche Beiträge an das BRP. Eine Umsatzänderung von $0.5 \%$ in der chemischen Industrie in Brig-Visp-Naters wirkt direkt fünfzigmal stärker auf das BRP als die gesamte Wirkung, welche einer Umsatzänderung von $2 \%$ in der Landwirtschaft in Brig-Visp-Naters folgt. Diese tatsächliche Wirksamkeit lässt sich ermitteln, indem prozentuale Wirkungen auf Regionalumsatz, BRP und Gesamtbeschäftigung bei einprozentigen Umsatzänderungen und unter Berücksichtigung der Multiplikatoren 


\begin{tabular}{|c|c|c|c|c|c|}
\hline \multirow[t]{2}{*}{ Branche } & des Bruttoregic & alprodukts in $\mathrm{Pr}$ & ent bei $\pm 1 \%$ & Umsatz in der jew & eiligen Branche \\
\hline & Val Bregaglia* & Val Müstair** & Urserntal** & Brig-Visp-Naters* & Vallemaggia** \\
\hline Landwirtschaft & 0.02 & 0.08 & 0.03 & 0.02 & 0.01 \\
\hline Bergbau & -- & -- & -- & -- & 0.10 \\
\hline $\begin{array}{l}\text { Nahrungsmittelproduktion } \\
\text { Nahrungsmittelhandel }\end{array}$ & 0.17 & 0.11 & 0.06 & 0.02 & $\begin{array}{l}0.01 \\
0.02\end{array}$ \\
\hline Gewerbe & 0.05 & 0.24 & 0.04 & 0.02 & 0.05 \\
\hline Chemische Industrie & -- & -- & -- & 0.33 & -- \\
\hline Bauwesen & 0.27 & 0.22 & 0.12 & 0.20 & 0.09 \\
\hline Energie, Wasser & 0.07 & 0.02 & 0.05 & 0.00 & 0.25 \\
\hline Handel, Reparatur & -- & 0.08 & -- & 0.29 & 0.03 \\
\hline $\begin{array}{l}\text { Tourismus } \\
\text { Gastgewerbe }\end{array}$ & 0.18 & 0.08 & $\begin{array}{l}0.47 \\
0.17\end{array}$ & 0.02 & 0.07 \\
\hline Sonstige Dienstleistungen & 0.03 & 0.15 & 0.04 & 0.28 & 0.11 \\
\hline Verkehr, Kommunikation & & & & 0.03 & \\
\hline Immobilien, Informatik & & & & 0.03 & \\
\hline Gesundheitswesen & - & & & & 0.08 \\
\hline Militär, Bundesstellen & -- & -- & 0.22 & -- & -- \\
\hline Öffentlicher Sektor & 0.20 & 0.25 & 0.09 & 0.05 & 0.34 \\
\hline
\end{tabular}

* ohne induzierte Effekte, nur indirekte Effekte

${ }^{* *}$ mit indirekten und induzierten Effekten

Tab. 2: Endogene Wachstumspotenziale der Branchen, in Prozent

Endogenous growth potential of various industries, in percent

Potentiels de croissance endogènes par branches, en pourcentage

Quelle: Zusammenzug aus BUSER 2005

berechnet werden. In Tabelle 2 sind diese sogenannten endogenen Wachstumspotenziale aufgelistet. Eine Umsatzänderung um $1 \%$ in der Landwirtschaft des Val Bregaglia verändert beispielsweise die gesamte regionale Bruttowertschöpfung um $0.02 \%$.

Aus Tabelle 2 wird ersichtlich, dass trotz des tiefen Multiplikators von 0.48 in Tabelle 1 die chemische Industrie in Brig-Visp-Naters mit einem endogenen Wachstumspotenzial von $0.33 \%$ den grössten Einfluss auf das regionale Wachstum hat. Brig-Visp-Naters ist folglich nicht nur ein Dienstleistungszentrum, sondern auch eine Industrieregion.

Dass nicht nur die effiziente Verflechtung in ein Wertschöpfungssystem, sondern auch die monetäre Bedeutung einer Branche zur Wirksamkeit beiträgt, zeigt sich neben der chemischen Industrie in BrigVisp-Naters in gleicher Weise auch an der Energieproduktion im Vallemaggia.

Angenommen grösstmögliches Wirtschaftswachstum sei das adäquate regionalpolitische Ziel, so verändern sich mit der Betrachtung der Wirksamkeit offenkundig wirtschaftspolitische Prioritäten. Vormals als effizient beurteilte Branchen werden wegen geringer Effektivität für regionalpolitische Interventionen unge- eignet. Wie sich zeigt, gilt dies in besonderem Masse für die Landwirtschaft. Abgeltungen im Rahmen der Multifunktionalität, welche mit dem bedeutenden gesamtwirtschaftlichen Beitrag der Landwirtschaft im Berggebiet begründet werden, müssen als effizient aber zu wenig wirksam kontrovers diskutiert werden.

Auch dem Tourismus in den Alpen kommt weniger Bedeutung zu als schlechthin angenommen. Von den untersuchten Regionen weist nur das Urserntal einen Tourismus auf, welcher genügend Potenzial für langfristiges Wachstum ausweist. Obschon sich das Val Müstair mit dem schweizweit einzigen Nationalpark als Tourismusregion versteht (KüPFER 2000), kann nicht genügend Wirtschaftswachstum herbeigeführt werden.

Die Wirksamkeit beschreibt, welche regionalen Auswirkungen tatsächlich von einer Umsatzänderung zu erwarten sind. Damit aber eine Branche die Umsätze steigern kann, braucht sie Zugang zu weiterwachsenden Märkten. Diese Eigenschaft haben nur Märkte ausserhalb der Berggebiete in den nationalen und internationalen Zentren. Entsprechender Marktzugang gelingt nur, wenn mit hoher Produktivität und Innovationsfähigkeit konkurrenzfähig angeboten werden kann. Zur Festsetzung regionalpolitischer Prio- 


\begin{tabular}{|lcccccr|}
\hline Branche & $\begin{array}{c}\text { Regionale } \\
\text { Produktion }\end{array}$ & $\begin{array}{c}\text { Private } \\
\text { Nachfrage }\end{array}$ & $\begin{array}{c}\text { Offentliche } \\
\text { Nachfrage }\end{array}$ & $\begin{array}{c}\text { Touristische } \\
\text { Nachfrage }\end{array}$ & $\begin{array}{c}\text { Exporte in } \\
\text { Umgebungs- } \\
\text { regionen }\end{array}$ & $\begin{array}{c}\text { Übrige } \\
\text { Exporte }\end{array}$ \\
\hline Landwirtschaft & 41.0 & 31.8 & 18.0 & 3.1 & 3.9 & 2.1 \\
Nahrungsmittel, Getränke & 26.4 & 57.2 & 1.5 & 6.9 & 7.0 & 1.0 \\
Handwerk & 49.8 & 6.7 & 3.3 & 0.5 & 3.9 & 35.7 \\
Chemische Industrie & 0.6 & 0.1 & 0.0 & 0.0 & 0.1 & 99.1 \\
Energie, Wasser & 43.4 & 34.6 & 3.7 & 0.6 & 6.8 & 10.9 \\
Bauwesen & 13.2 & 22.2 & 9.7 & 0.1 & 46.6 & 8.1 \\
Handel, Reparatur & 28.1 & 10.2 & 1.1 & 0.7 & 28.5 & 31.6 \\
Hotel-, Gastgewerbe & 5.6 & 31.6 & 0.3 & 60.0 & 1.0 & 1.5 \\
Verkehr, Kommunikation & 28.4 & 9.2 & 6.0 & 1.2 & 13.0 & 42.2 \\
Immobilien, Informatik & 33.3 & 11.5 & 5.0 & 0.8 & 18.8 & 22.4 \\
Sonstige Dienstleistungen & 31.6 & 19.6 & 4.3 & 0.2 & 23.9 & 20.3 \\
Öffentlicher Sektor & 18.3 & 3.0 & 73.0 & 0.7 & 2.6 & 2.3 \\
Regionale Wertschöpfung & $\mathbf{1 9 . 4}$ & $\mathbf{1 1 . 7}$ & $\mathbf{5 . 3}$ & $\mathbf{1 . 0}$ & $\mathbf{1 7 . 9}$ & $\mathbf{4 4 . 5}$ \\
\hline
\end{tabular}

Tab. 3: Absolute Wertschöpfungsabhängigkeiten für Brig-Visp-Naters, in Prozent (ohne induzierte Effekte, nur indirekte Effekte)

Absolute dependencies for value added in the region of Brig-Visp-Naters, in percent (without induced effects, only indirect effects)

Dépendances absolues de création de valeur, selon «Brig-Visp-Naters», en pourcentage (sans effets induits, uniquement effets indirects)

Quelle: BUSER 2005: 175 (verändert)

ritäten müssen bestehende und mögliche Markteintritte in wachsende Exportmärkte geprüft werden. Der Input-Output-Ansatz stellt auch hierfür eine Methode zur Verfügung, wobei gegenüber den Multiplikatoren Wertschöpfungsketten und Wertschöpfungssysteme in «umgekehrter» Sichtweise analysiert werden. Daraus lassen sich absolute Abhängigkeiten von Umsatz, Wertschöpfung, Beschäftigung und Einkommen berechnen. Diese absoluten Wertschöpfungsabhängigkeiten geben prozentual an, welcher Anteil der Wertschöpfung einer Branche beziehungsweise einer Region durch die Nachfrage in den unterschiedlichen Absatzkanälen entsteht. Dabei kann die Wertschöpfung durch direkte, indirekte oder auch induzierte Nachfrage entstehen.

Die absoluten Wertschöpfungsabhängigkeiten für BrigVisp-Naters zeigen, wie limitiert die regionale Nachfrage im Berggebiet ist (Tabelle 3). Knapp 20\% der Wertschöpfung entstehen durch regionale Belieferung mit Vorleistungen. Etwa 12\% gehen auf den Konsum der privaten Haushalte zurück, die öffentlichen Haushalte lösen weitere 5 bis $6 \%$ des BRP aus. Am weitaus meisten Wertschöpfung geht mit $45 \%$ auf den Export von Gütern und Dienstleistungen in den Rest der Welt zurück. Der Anteil der Branchenwertschöpfung aus Exporten über Umgebungsregionen hinaus beträgt zwischen 1 und $99 \%$. 17 bis $19 \%$ der regionalen Wertschöpfung gehen auf Exporte in die umgebenden
Regionen zurück, wo zurzeit auch die Grossbaustelle des NEAT-Lötschbergbasistunnels beliefert wird.

Klassische Entleerungsregionen wie auch die zentrumsnahe Transformationsregion zeigen keine grundsätzlichen Abweichungen zum untersuchten Zentrum. Innerhalb der regionalen Nachfrage gibt es unbedeutende Verschiebungen weg vom regionalen Produktionssystem hin zur privaten und öffentlichen Nachfrage. In keinem Fall löst die regionale Nachfrage aber mehr als $40 \%$ der Wertschöpfung aus. Für sämtliche Untersuchungsregionen zeigt sich, dass 45 bis $50 \%$ der regionalen Wertschöpfung durch Exporte in nationale und internationale Zentren zustande kommen. Tourismus und Lieferungen in angrenzende regionale Zentren lösen die restlichen 15 bis $20 \%$ des BRP aus. Langfristiges Wirtschaftswachstum ist nur noch über Exporte in nationale und internationale Zentren herbeizuführen. Hierzu zählt auch die Leistungserbringung an Touristen aus diesen Zentren. Alle weiteren Absatzkanäle sind in der Nachfragekapazität begrenzt.

\section{Fazit: Wege zum langfristigen Wirtschaftswachstum}

Aus Wirksamkeit und Exportmöglichkeiten lässt sich ein einfaches wachstumspolitisches Modell herleiten, 


\begin{tabular}{|c|c|c|c|c|c|}
\hline & Region & Knotenpunkte & Wachstumspole & Autonome Branchen & Mitläufer-Branchen \\
\hline \multirow{3}{*}{ 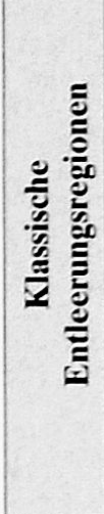 } & Val Bregaglia & $\begin{array}{l}\text { Bauwesen } \\
\text { Öffentlicher } \\
\text { Sektor }\end{array}$ & Energie & $\begin{array}{l}\text { Nahrungsmittel } \\
\text { Dienstleistungen } \\
\text { Tourismus }\end{array}$ & $\begin{array}{l}\text { Landwirtschaft } \\
\text { Gewerbe }\end{array}$ \\
\hline & Val Müstair & $\begin{array}{l}\text { Gewerbe } \\
\text { Öffentlicher } \\
\text { Sektor }\end{array}$ & Bauwesen & $\begin{array}{l}\text { Handel } \\
\text { Nahrungsmittel } \\
\text { Tourismus }\end{array}$ & $\begin{array}{l}\text { Landwirtschaft } \\
\text { Energie } \\
\text { Dienstleistungen }\end{array}$ \\
\hline & Urserntal & keine & $\begin{array}{l}\text { Tourismus } \\
\text { Gastgewerbe } \\
\text { Militär, Bund }\end{array}$ & keine & $\begin{array}{l}\text { Landwirtschaft } \\
\text { Nahrungsmittel } \\
\text { Gewerbe } \\
\text { Bauwesen } \\
\text { Energie } \\
\text { Dienstleistungen } \\
\text { Öffentlicher Sektor }\end{array}$ \\
\hline 龍 & $\begin{array}{l}\text { Brig-Visp- } \\
\text { Naters }\end{array}$ & $\begin{array}{l}\text { Sonstige Dienst- } \\
\text { leistungen }\end{array}$ & $\begin{array}{l}\text { Chemische } \\
\text { Industrie } \\
\text { Bauwesen } \\
\text { Handel, } \\
\text { Reparatur }\end{array}$ & $\begin{array}{l}\text { Hotel-/Gastgewerbe } \\
\text { Verkehr, } \\
\text { Kommunikation } \\
\text { Immobilien, } \\
\text { Informatik } \\
\text { Dienstleistungen }\end{array}$ & $\begin{array}{l}\text { Landwirtschaft } \\
\text { Nahrungsmittel } \\
\text { Handwerk } \\
\text { Energie, Wasser } \\
\text { Öffentlicher Sektor }\end{array}$ \\
\hline 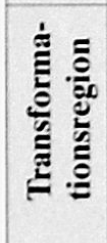 & Vallemaggia & $\begin{array}{l}\text { Dienstleistungen } \\
\text { Öffentlicher } \\
\text { Sektor }\end{array}$ & $\begin{array}{l}\text { Bergbau } \\
\text { Energie }\end{array}$ & $\begin{array}{l}\text { Landwirtschaft } \\
\text { Handwerk }\end{array}$ & $\begin{array}{l}\text { Nahrungsmittelproduktion } \\
\text { Nahrungsmittelhandel } \\
\text { Handel, Reparatur } \\
\text { Bauwesen } \\
\text { Tourismus } \\
\text { Gesundheitswesen }\end{array}$ \\
\hline
\end{tabular}

Tab. 4: Wachstumsportfolios der Untersuchungsregionen Growth portfoli for the regions under investigation Portfolios de croissance des régions soumises a l'étude Quelle: Buser 2005: 225

welches auf regionalpolitische Prioritäten hinweist. Das Modell wird regionales Wachstumsportfolio genannt. Es berechnet, ob Wirksamkeit und Exporttätigkeiten von Branchen über- oder unterdurchschnittlich sind. Als Kennziffer für die Wirksamkeit gelten die endogenen Wachstumspotenziale, die Exporttätigkeit wird mittels der absoluten Wertschöpfungsabhängigkeit von Exporten quantitativ operationalisiert. Jeder der vier Fälle fasst die Branchen zusammen. welche gleiche Eigenschaften für die regionale Wachstumspolitik aufweisen. Tabelle 4 zeigt die Wachstumsportfolios für die Untersuchungsregionen, wobei die Fälle beschreibend benannt sind.

Langfristiges Wirtschaftswachstum wird durch die Wachstumspole ausgelöst. Knotenpunkte und autonome Branchen sind potenziclle Wachstumspole. welche jedoch Schwächen bezüglich externem Marktzutritt oder Wirksamkeit aufweisen. Im Soll-Zustand werden diese Schwächen überwunden und die entsprechenden Branchen an die Funktion eines Wachstumspoles herangeführt. Nicht jede Zunahme von Wirksamkeit und Exporttätigkeit führt jedoch Wachs- tumspole herbei. da sich mit einzelnen Branchen immer auch gesamte regionale Wirtschaftsgefüge verändern. Würde unvermittelt eine enorm umsatzund wertschöpfungsstarke Unternehmung neu auftreten. könnte dies ein regionales Wachstumsportfolio beispielsweise so stark ändern. dass nur noch eine Branche als Wachstumspol gilt.

Ein regionales Wachstumsportfolio kann also als IstZustand verstanden werden. welcher Schwächen und Wege hin zum wachstumsmaximalen Soll-Zustand aufzeigt. Im Portfolio verfügen nur Wachstumspole über überdurchschnittliche Exportaktivität und Wirksamkeit. Knotenpunkte und autonome Branchen sind potenzielle Wachstumspole. weisen aber Schwächen wie geringe Wirksamkeit oder fehlenden Marktzugang auf. Eine Region sichert sich langfristig stabiles Wachstum. wenn möglichst viel Wertschöpfung in den Wachstumspolen generiert wird. Mit steigender Anzahl Wachstumspole nimmt die Stabilität des Wirtschaftswachstums zu. Gleiches gilt. wenn die Anzahl bedienter Märkte zunimmt (siehe beispielsweise Brig-Visp-Naters). Die Tabelle 4 zeigt aller- 
dings, dass in Entleerungsregionen allzu oft eine einzelne grosse Unternehmung als Wachstumspol wirkt. Elektrizitätsmarktordnungen und Eindämmung eines Baubooms in touristischen Regionen können als politisch gegebene Rahmenbedingungen entsprechend kurzfristig regionale Stagnation und Rezession auslösen, längerfristig können sich Wachstumschancen hierdurch allerdings auch verbessern.

Die Aufgabe der Regionalpolitik besteht darin, bestehende Wachstumspole zu stärken und potenzielle Wachstumspole bei der Überwindung bestehender Schwächen zu unterstützen. Für Mitläufer-Branchen gilt es, vermehrten Marktzugang zu Exportmärkten zu schaffen, ohne dabei aber Wettbewerbsverzerrungen zu produzieren. Innovationen in Produkt, Leistungen und Produktion unterstützen die für erfolgreichen Marktzugang notwendige Konkurrenzfähigkeit ganz gezielt. Autonome Branchen müssen einerseits Marktanteile ausbauen und anderseits regionale höhere Wirksamkeit aufweisen. Höhere Wirksamkeit ergibt sich dann, wenn der Rückgriff auf starke regionale Wertschöpfungssysteme möglich ist. Da sich erwiesenermassen regional sehr unterschiedliche Ist-Zustände zeigen, müssen die entsprechenden Strategien und Prioritäten immer im regionalen Kontext definiert werden. Die Anbindung an nationale und internationale Zentren ist trotz regionaler Ausgestaltung nicht aus den Augen zu verlieren. Die Vision der Expertenkommission von einer neuen Regionalpolitik kann mit der vorliegenden empirischen Untersuchung als zukunftsträchtig bezeichnet werden.

\section{Literatur}

BuSER, B. (2005): Regionale Wirtschaftskreisläufe und regionale Wachstumspolitik. Regionalpolitische Prioritäten für unterschiedliche Regionen im Schweizer Alpenraum auf der Basis regionaler Input-Output Tabellen. $-=$ Berichte aus der Volkswirtschaft, Aachen: Shaker Verlag.

EXPERTENKOMMISSION «ÜBERPRÜFUNG UND NEUKONZEPTION DER REgIONALPOLITIK» (2003): Neue Regionalpolitik (NRP). Schlussbericht. - = Expertenbericht im Auftrag des Staatssekretariats für Wirtschaft seco, Bern.

FurRer, H.-P. (2004): Unterschiedliche Verfahren zur Erstellung von regionalen Input-Output-Tabellen. Unveröffentlichte Diplomarbeit, Geographisches Institut, Universität Zürich.

Hahne, U. \& K. von Stackelberg (1994): Regionale Entwicklungstheorien. Konkurrierende Ansätze zur Erklärung der wirtschaftlichen Entwicklung in Regionen. - Freiburg: EURES Institut für regionale Studien in Europa.

Holub, H.-W.\& H.Schnabl (1994): Input-Output-Rechnung. Input-Output Tabellen. Einführung. $-=$ Olden- bourgs Lehr- und Handbücher der Wirtschafts- und Sozialwissenschaften, 3. Auflage, München: Oldenbourg. KOPAINSKY, B. (2005): A system dynamics analysis of socio-economic development in lagging Swiss regions. - = Berichte aus der Volkswirtschaft, Aachen: Shaker Verlag.

KüPFER, I. (2000): Die regionalwirtschaftliche Bedeutung des Nationalparktourismus, untersucht am Beispiel des Schweizerischen Nationalparks. $-=$ Nationalpark-Forschung in der Schweiz 90. Zernez.

Menghetti, P. \& A. Zimmermann (1996): Makroökonomie. - Winterthur: Verlag Hans Schellenberg.

OECD (2002): OECD Prüfbericht Raumentwicklung Schweiz. - Paris: OECD.

Rieder, P., Buchli, S. \& B. Kopainsky (2004): Erfüllung des Verfassungsauftrages durch die Landwirtschaft unter besonderer Berücksichtigung ihres Beitrags zur dezentralen Besiedlung. - = Report zuhanden des Bundesamtes für Landwirtschaft, Bern, Zürich: Institut für Agrarwirtschaft der ETH Zürich.

Thierstein, A. \& H. Behrendt (2001): Überprüfung der Zielerreichung der Schweizer Regionalpolitik. - = Report zuhanden des Staatssekretariats für Wirtschaft (seco), Bern, Zürich: Institut für Orts-, Regional- und Landesplanung der ETH Zürich.

\section{Zusammenfassung: Festsetzung regionalpolitischer Prioritäten im Schweizer Alpenraum auf der Basis regionaler Input-Output-Tabellen - ein Überblick} Die einzelnen Regionen der Schweiz erfahren immer weniger Wirtschaftswachstum. Gleichzeitig nehmen Disparitäten zwischen Zentren und peripheren Berggebieten weiter zu. Die bisherige regionale Wirtschaftspolitik hat keine Nivellierung von Wachstum und Einkommen bewirkt. Zur Ausgestaltung einer neuen Regionalpolitik sind daher detaillierte Kenntnisse über regionale Wirtschaftskreisläufe notwendig. Mittels regionaler Input-Output-Tabellen werden für fünf Regionen Wirtschaftskreisläufe mathematisch modelliert. Aus den Modellen können mittels Nachfragemultiplikatoren regionalwirtschaftliche Effizienzen, Effektivitäten und Abhängigkeiten berechnet werden. Zusammengefügt in einem Wachstumsportfolio lässt sich auf regionale wachstumspolitische Prioritäten schliessen. Regionen können langfristig nur wachsen, wenn ein überdurchschnittlicher Zugang zu den stetig wachsenden Märkten der Zentren besteht. Regionalpolitik muss immer externen Marktzugang und regionale Wirksamkeit fördern.

\section{Summary: Prioritisation of regional policy in the Swiss Alps using regional input-output tables - an overview}

Economic growth in Swiss regions is decreasing while disparities between urban centres and peripheral mountain valleys have grown. Current regional eco- 
nomic policy has not managed to obtain an equilibrium between growth and income. In order to set up a new regional policy, detailed knowledge of regional economic cycles is essential. Using regional inputoutput tables economic cycles have been mathematically modelled for five regions. As a result, economic efficiencies, effectiveness and economic dependencies within the regions can be shown by way of demand multipliers. Merging all in a so-called regional growth portfolio, priorities for regional growth strategies can be defined. It is maintained that regions can only grow in the long term by assuring access to the growth markets of urban centres at an above-average level. Further, regional policy should aim to promote both external market access and regional effectiveness simultaneously.

\section{Résumé: Détermination des priorités en matière de politique régionale dans l'arc alpin suisse, sur la base des tableaux de l'input-output régional}

La croissance économique des diverses régions suisses se réduit constamment. En même temps, les disparités entre les centres et les territoires périphériques de montagne continuent à augmenter. La politique économique régionale mise en œuvre jusqu'à présent n'a pas suscité l'ajustement attendu entre la croissance et le revenu. C'est pourquoi l'élaboration d'une nouvelle politique régionale requiert des connaissances détaillées sur les circuits économiques. A l'appui de tableaux régionaux d'input-output, la présente contribution se livre à un modèle mathématique pour cinq régions. Les modèles permettent de déterminer, à l'aide de multiplicateurs de la demande, des dépendances dans le domaine de l'efficience économique régio- nale et de l'efficacité. Le portfolio global de croissance, élaboré à cet effet, témoigne de la nécessité de déterminer des priorités politiques de croissance régionale. Les régions ne peuvent bénéficier d'une croissance à long terme que lorsqu'elles ont de manière permanente un accès, supérieur à la moyenne, aux marchés centraux. La politique régionale doit promouvoir constamment l'accès externe au marché et l'efficacité régionale.

Dr. Benjamin Buser, Avenir Suisse, Giessereistrasse 5, $\mathrm{CH}-8005$ Zürich.

e-mail:benjamin.buser@avenir-suisse.ch

Dr. Gianluca Giuliani, Fondazione movingAlps, c/o Institut für Agrarwirtschaft, ETH-Zentrum (SOL), Sonneggstrasse 33, CH-8092 Zürich.

e-mail: gianluca.giuliani@iaw.agrl.ethz.ch

Prof. Dr. Peter Rieder, Institut für Agrarwirtschaft, ETH-Zentrum (SOL), Sonneggstrasse 33, CH-8092 Zürich.

e-mail: peter.rieder@iaw.agrl.ethz.ch

\section{Manuskripteingang/received/manuscrit entré le 30.6.2005}

Annahme zum Druck/accepted for publication/accepté pour l'impression: 16.12.2005 